\title{
Linear System Identification as Curve Fitting
}

\author{
Lennart Ljung, \\ Division of Automatic Control \\ Department of Electrical Engineering \\ Linköpings universitet, SE-581 83 Linköping, Sweden \\ WWW: http://www. control.isy.liu.se \\ E-mail: Ljung@isy.liu.se, @isy.liu.se
}

3rd December 2003

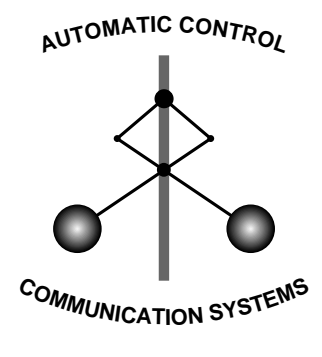

LINKÖPING

Report no.: LiTH-ISY-R-2466

Submitted to Spinger Lecture Notes on Control and Information, Vol 286: 203-215 2003//Conference in honor of Anders Lindquist

Technical reports from the Control \& Communication group in Linköping are available at http://www. control.isy.liu.se/publications. 
Linear System Identification as Curve Fitting

Lennart Ljung

August 7, 2002 


\title{
Chapter 1
}

\section{Linear System Identification as Curve Fitting}

\begin{abstract}
The purpose of this contribution is to point out and exploit the kinship between identification of linear dynamic systems and classical curve fitting. For curve fitting we discuss both global and local parametric methods as well as non-parametric ones, such as local polynomial methods. We view system identification as the estimation of the frequency function curve. The empirical transfer function estimate is taken as the "observations" of this curve. In particular we discuss how this could be done for multi-variable systems. Local and non-parametric curve fitting methods lead to variants of classical spectral analysis, while the prediction error/maximum likelihood framework corresponds to global parametric methods. The role of the noise model in dynamic models is also illuminated from this perspective.
\end{abstract}

\section{$1.1 \quad$ Introduction}

A linear dynamic system in discrete or continuous time can be described by

$$
y(t)=G(\sigma) u(t)+v(t)
$$

where $\sigma$ is the differentiation operator $p$ in continuous time and the shift operator $q$ in discrete time. The identification problem is to find the transfer operator $G$ and possibly also the spectrum of the additive noise $v$. There is an extensive literature on this problem, see among many books, e.g. [7] and $[11]$. 
One may distinguish three main approaches to this problem:

- Spectral Analysis which forms estimates of the spectra of the output, the input and the cross spectrum between input and output, $\Phi_{y}(\omega), \Phi_{u}(\omega)$, $\Phi_{y u}(\omega)$ and then the estimates

$$
\begin{aligned}
\hat{G}(i \omega) & =\frac{\Phi_{y u}(\omega)}{\Phi_{u}(\omega)} \\
\hat{\Phi}_{v}(\omega) & =\Phi_{y}(\omega)-\hat{G}(i \omega) \Phi_{u}(\omega)
\end{aligned}
$$

See, e.g. [5], [1] for thorough treatments of this approach.

- Parametric Methods that explicitly parameterize the sought transfer functions and estimate the parameters by maximum likelihood or related techniques.

- Subspace Methods that conceptually can be described as model reduction schemes of simple high order estimates. See, e.g. [14], [6] or [15].

It is the purpose of this contribution to put such identification methods into the perspective of simple-minded curve fitting techniques. To put it another way: We are not so far from Gauss' basic contributions on least squares.

\subsection{Curve Fitting}

To bring out the basic features of the estimation problem, let us study a simple example. Suppose the problem is to estimate an unknown function $g_{0}(x),-1 \leq x \leq 1$. The observations we have are noise measurements $y(k)$ at points $x_{k}$ which we may or may not choose ourselves:

$$
y(k)=g_{0}\left(x_{k}\right)+e(k)
$$

How to approach this problem?

\subsubsection{Parametric Methods}

\section{Global Parameterizations}

One way or another we must decide "where to look for" $g$. We could, for example, have the information that $g$ is a third order polynomial. This would lead to the - in this case - grey box model structure

$$
g(x, \theta)=\theta_{1}+\theta_{2} x+\theta_{3} x^{2}+\ldots+\theta_{n} x^{n-1}
$$


with $n=4$, and we would estimate the parameter vector $\theta$ from the observations $y$, using for example the classical least squares method.

Now suppose that we have no structural information at all about $g$. We would then still have to assume something about it, e.g. it is an analytical function, or that it is piecewise constant or something like that. In this situation, we could still use (1.5), but now as black-box model: if we assume $g$ to be analytic we know that it can be approximated arbitrarily well by a polynomial. The necessary order $n$ would not be known, and we would have to find a good value of it using some suitable scheme.

Note that there are several alternatives in this black-box situation: We could use rational approximations:

$$
g(x, \theta)=\frac{\theta_{1}+\theta_{2} x+\theta_{3} x^{2}+\ldots+\theta_{n} x^{n-1}}{1+\theta_{n+1} x+\theta_{n+2} x^{2}+\ldots+\theta_{n+m-1} x^{m-1}}
$$

or Fourier series expansions

$$
g(x, \theta)=\theta_{0}+\sum_{\ell=1}^{n} \theta_{2 \ell-1} \cos (\ell \pi x)+\theta_{2 \ell} \sin (\ell \pi x)
$$

\section{Local Parameterizations}

Alternatively, we could approximate the function by piece-wise constant functions, as illustrated in Figure 1.1. The mapping $g$ can be parameterized as a

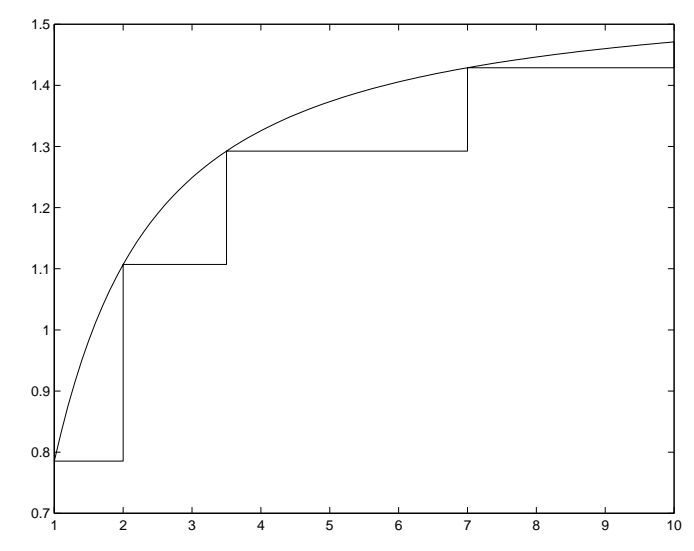

Figure 1.1: A piece-wise constant approximation. 
function expansion

$$
g(x, \theta)=\sum_{k=1}^{n} \alpha_{k} \kappa\left(\beta_{k}\left(x-\gamma_{k}\right)\right)
$$

Here, $\kappa$ is a "mother basis function", from which the actual functions in the function expansion are created by dilation (parameter $\beta$ ) and translation (parameter $\gamma$ ). For example, with $\kappa=\cos$ we would get Fourier series expansion with $\beta$ as frequency and $\gamma$ as phase. More common are cases where $\kappa$ is a unit pulse. With that choice, (1.8) can describe any piecewise constant function, where the granularity of the approximation is governed by the dilation parameter $\beta$. Compared to Figure 1.1 we would in that case have

- $n=4$,

- $\gamma_{1}=1, \gamma_{2}=2, \gamma_{3}=3.5, \gamma_{4}=7$,

- $\beta_{1}=1, \beta_{2}=2 / 3, \beta_{3}=1 / 3.5, \beta_{4}=1 / 3$

- $\alpha_{1}=0.79, \alpha_{2}=1.1, \alpha_{3}=1.3, \alpha_{4}=1.43$

A related choice is a soft version of a unit pulse, such as the Gaussian bell. Alternatively, $\kappa$ could be a unit step (which also gives piecewise constant functions), or a soft step, such as the sigmoid.

\subsubsection{Estimation Techniques and Basic Properties}

It suggests itself that the basic least-squares like approach is a natural approach for curve fitting:

$$
\begin{aligned}
\hat{\theta}_{N} & =\arg \min _{\theta} V_{N}(\theta) \\
V_{N}(\theta) & =\sum_{k=1}^{N} \mu_{k}\left(y(k)-g\left(x_{k}, \theta\right)\right)^{2}
\end{aligned}
$$

Here $\mu_{k}$ is a weight that in a suitable way reflects the

- "reliability" of the measurement $k$. This is typically evaluated as the variance of $e(k)$, so we would have $\mu_{k} \sim 1 / \lambda_{k}$, where $\lambda_{k}$ is the variance of $e(k)$.

- "relevance" of the measurement $k$. It could be that we do not fully believe that the underlying model $g(x, \theta)$ is capable of describing the data for all $x$. We could then downweigh a measurement at a point $x_{k}$ outside a region of prime relevance for the model. 
In case $y$ and $g_{0}$ are vectorvalued (column vectors), the criterion takes the form

$$
V_{N}(\theta)=\sum_{k=1}^{N}\left(y(k)-g\left(x_{k}, \theta\right)\right)^{T} \Lambda_{k}^{-1}\left(y(k)-g\left(x_{k}, \theta\right)\right.
$$

where the matrix $\Lambda_{k}$ takes care of the weightings. For the reliablity aspect, $\Lambda_{k}$ would be the covariance matrix of $e(k)$.

\subsubsection{Non-Parametric Methods}

\section{Basic Idea: Local Smoothing}

A simple idea to form an estimate of the function value $g(x)$ at a point $x$ is to form some kind of average of the observations $y(k)$ corresponding to $x_{k}$ in the neigbourhood of $x$ :

$$
\hat{g}(x)=\sum_{k=1}^{N} W\left(x, x_{k}\right) y(k)
$$

where the weights $W$ are chosen appropriately, and typically being zero when the distance between $x$ and $x_{k}$ is larger than a certain value ("the bandwidth"). The choice of such weights is the subject of an extensive literature in statistics. See, among many references, e.g. [3], [13], [2], and [9]. It is not the purpose of this paper to give an overview of such methods, but we can point to some basic choices.

\section{Nearest Neighbor Smoothing}

Perhaps the simplest idea is to take as an estimate of $\hat{g}$ the observed value $y(k)$ at the nearest observation point. This corresponds to choosing $W\left(x, x_{k}\right)$ to be 1 for that $x_{k}$ in the observation set that is closest to $x$ and 0 otherwise.

\section{Kernel Smoothers}

Take

$$
W(x, z)=\kappa(|x-z| / h)
$$

where $\kappa(\xi)$ is some bell-shaped function that is zero for $\xi>1$. A common choice is the Epanechnikov kernel

$$
\kappa(x)= \begin{cases}1-x^{2} & \text { for }|x|<1 \\ 0 & \text { for }|x| \geq 1\end{cases}
$$




\section{Local Polynomial Methods}

Polynomial approximations of a curve may be efficient, at least locally. Instead of the global model (1.5) one may seek a local approximation in the neigbourhood of $x$ by

$$
g(z, \theta)=\theta_{1}+\theta_{2}(z-x)+\theta_{3}(z-x)^{2}+\ldots \theta_{n}(z-x)^{n-1}
$$

Then estimate these coefficients e.g. by weighted least squares

$$
\min \sum_{k=1}^{N} \mu_{k} \cdot \tilde{W}\left(x-x_{k}\right) \cdot\left|y(k)-\theta_{1}-\theta_{2}\left(x_{k}-x\right)-\ldots-\theta_{n}\left(x_{k}-x\right)^{n-1}\right|^{2}
$$

Here $\mu_{k}$ is a weight that reflects the reliability of the $k$ :th measurement (the variance of $e(k))$ and $\tilde{W}$ concentrates the fit to a suitable neighbourhood of $x$. The resulting estimate $\hat{\theta}$ will be linear in $y(k)$ and choosing $\hat{\theta}_{1}$ as an estimate for $g(x)$ will give an expression of the type (1.11).

\section{Direct Optimization of Weights}

In a recent approach, [10], the choice of weights $W$ in (1.11) is formulated as a min-max optimization problem for the mean square error $\mathrm{E}|g(x)-\hat{g}(x)|^{2}$. This is either a quadratic programming problem or a convex one and can be solved quite efficiently to achieve optimal weights without relying upon asymptotic theory.

\subsection{Linear Dynamic Models}

\subsubsection{The Curve: The Frequency Function}

A linear dynamic system is uniquely defined, e.g., by its impulse response or by its Frequency Function

$$
G\left(e^{i \omega T}\right) \quad \text { or } \quad G(i \omega)
$$

in discrete time, or continuous time, respectivly. For simplicity in the sequel we will use the continuous time notation.

Therefore it is possible to interpret all methods for linear system identification as methods to estimate the frequency function curve. 


\subsubsection{The Observations: The ETFE}

The primary observations from a dynamical system are always the sequences of sampled inputs and outputs, $u$ and $y$,

$$
Z^{N}=\left\{u\left(t_{1}\right), y\left(t_{1}\right), \ldots, u\left(t_{N}\right), y\left(t_{N}\right)\right\}
$$

From these we may form the Fourier transforms

$$
\begin{aligned}
& U_{N}(\omega)=\frac{1}{\sqrt{N}} \sum_{k=1}^{N} u\left(t_{k}\right) e^{i \omega(k-1) T} \\
& Y_{N}(\omega)=\frac{1}{\sqrt{N}} \sum_{k=1}^{N} y\left(t_{k}\right) e^{i \omega(k-1) T}
\end{aligned}
$$

(These expressions give the DFT for equidistantly sampled data: $t_{k+1}-t_{k} \equiv T$, but several variants can be envisioned.)

For a scalar input we may now form the Empirical Transfer Function Estimate, ETFE as

$$
\hat{\hat{G}}_{N}(i \omega)=\frac{Y_{N}(\omega)}{U_{N}(\omega)}
$$

In case the observations $y$ and $u$ have been obtained from a noise-corrupted linear system with frequency function $G_{0}(i \omega)$ it can be shown that the ETFE has the following statistical properties: (Lemma 6.1 in [7].)

$$
\begin{array}{r}
\mathrm{E} \hat{\hat{G}}_{N}(i \omega)=G_{0}(i \omega)+\frac{\rho_{1}}{\sqrt{N} U_{N}(\omega)} \\
\mathrm{E}\left|\hat{\hat{G}}_{N}(i \omega)-G_{0}(i \omega)\right|^{2}=\frac{\Phi_{v}(\omega)}{\left|U_{N}(\omega)\right|^{2}}+\frac{\rho_{2}}{N\left|U_{N}(\omega)\right|^{2}}
\end{array}
$$

Here $\Phi_{v}(\omega)$ is the spectrum of the additive noise (at the output of the system)and $\rho_{i}$ are constant bounds that depend on the impulse response of the system, the bound on the input, and the covariance function of the noise. Moreover, it can be shown that the EFTE's are asymptotically uncorrelated at frequencies on the DFT grid.

All this means that we can think of the ETFE as a "noisy measurement" of the frequency function:

$$
\hat{\hat{G}}_{N}\left(i \omega_{k}\right)=G_{0}\left(i \omega_{k}\right)+v_{k}
$$


with $v_{k}$ being a zero mean random variable with variance $\Phi_{v}\left(\omega_{k}\right) /\left|U_{N}\left(\omega_{k}\right)\right|^{2}$. We have then ignored the terms with $\rho$ in the expressions above.

Something must also be said about the frequency grid in (1.23): If the Fourier transforms are obtained by DFT of equidistantly sampled data, the natural frequencies to use in (1.23) are the DFT grid:

$$
\omega_{k}=k \pi / N ; \quad k=0, \ldots, N-1
$$

This gives two advantages:

- Frequencies in between these carry no extra information: they are merely (trigonometric) interpolations of the values on the DFT grid. This also determines the maximum frequency resolution of the frequency function.

- $v_{k}$ are (asymptotically) uncorrelated on this grid.

In the case of $p$ outputs, $v_{k}$ is a column vector and $\Phi_{v}$ is a $p \times p$ matrix.

\subsubsection{The Multi-input Case}

When there are several inputs, so that $u$ is an $m$-dimensional column vector and $G$ is a $p \times m$ matrix, there is no unique way of forming the ETFE, and this has apparently not been discussed in the literature. One of the possibilities is to split the data record into $m$ parts, like (assume $N=m M$ and $t_{k+1}-t_{k} \equiv 1$ for simplicity)

$$
U_{N}^{(r)}(\omega)=\frac{1}{\sqrt{M}} \sum_{k=1}^{M} u((r-1) M+k) e^{i \omega(k-1)} ; \quad r=1, \ldots, m
$$

and similarly for $Y_{N}^{(r)}$. The corresponding DFT-grid for $\omega$ will be reduced by a factor $m$ to

$$
\omega_{\ell}=\ell \pi / M ; \ell=0, \ldots, M-1
$$

On this grid we can define

$$
\begin{aligned}
\hat{\hat{G}}_{N}(i \omega) & =\left[\begin{array}{lll}
Y_{N}^{(1)}(\omega) & \ldots & Y_{N}^{(m)}(\omega)
\end{array}\right]\left[\begin{array}{lll}
U_{N}^{(1)}(\omega) & \ldots & U_{N}^{(m)}(\omega)
\end{array}\right]^{-1} \\
& =\mathcal{Y}_{N}(\omega) \mathcal{U}_{N}(\omega)^{-1}
\end{aligned}
$$

provided the $m \times m$ inverse exists (which is the generic case). A related possibility is to take DFTs of the whole data record and form the estimate using $m$-tuples of neighboring frequencies. 
It can be shown, analogously to the single input case that

$$
\hat{\hat{G}}_{N}\left(i \omega_{k}\right)=G_{0}\left(i \omega_{k}\right)+v_{k}
$$

where now $v_{k}$ is a sequence of $p \times m$ matrices with (asymptotically) zero means and asymptotically uncorrelated on the DFT-grid (1.26) with covariance matrix

$$
\left[\mathcal{U}_{N}(\omega) \mathcal{U}_{N}^{*}(\omega)\right]^{-1} \otimes \Phi_{v}(\omega)
$$

for $\operatorname{vec}\left(v_{k}\right)$. Here $\otimes$ denotes the Kronecker product, and vec means stacking the columns of a matrix on top of each other.

\subsection{Fitting the Frequency Function Curve by Local Methods}

The basic relation between the Fourier data and the frequency function is

$$
Y\left(\omega_{k}\right)=G\left(i \omega_{k}\right) U\left(\omega_{k}\right)+V\left(\omega_{k}\right), \quad k=1, \ldots, N
$$

This holds in the general multi-variable case. The covariance matrix for $V(\omega)$ is the $m \times m$ spectrum matrix $\Phi(\omega)$. For compact notation we form the $p \times N$ matrix $\mathcal{Y}$ as

$$
\mathcal{Y}=\left[Y\left(\omega_{1}\right), \ldots, Y\left(\omega_{N}\right)\right]
$$

and similarly the $m \times N$ matrix $\mathcal{U}$ and the $p \times N$ matrix $\mathcal{V}$. Then, if $G$ were a constant (complex valued) $p \times m$ matrix (1.30) could be rewritten

$$
\mathcal{Y}=G \mathcal{U}+\mathcal{V}
$$

This could be compared to the local polynomial approach (1.14) where we have only taken the constant term $(n=1)$. To estimate this constant $G$ we would apply weighted least squares (1.15) where the weighting function $\tilde{W}\left(\omega_{k}-\omega\right)$ would measure the distance between the value $\omega$, where we seek an estimate of $G$ and the frequencies $\omega_{k}$ where we have the observations. Form the $N \times N$ matrix $\mathcal{W}$ as the diagonal, real-valued matrix of these weights. Then the weighted least squares estimate of $G$ is

$$
\hat{G}=\mathcal{Y} \mathcal{W U}^{*}\left[\mathcal{U W U}^{*}\right]^{-1}
$$


This is the estimate at frequency $\omega$ and the dependence on $\omega$ in this expression is hidden in $\mathcal{W}$.

To estimate the disturbance spectrum $\Phi_{v}(\omega)$ we estimate $\mathcal{V}$ by

$$
\hat{\mathcal{V}}=\mathcal{Y}-\hat{G} \mathcal{U}=\mathcal{Y}\left(I-\mathcal{W} \mathcal{U}^{*}\left[\mathcal{U W U}^{*}\right]^{-1} \mathcal{U}\right)=\mathcal{Y} P_{u}
$$

where the last step is a definition of $P_{u}$. Note that

$$
P_{u} \mathcal{W} P_{u}^{*}=P_{u} \mathcal{W}
$$

A natural estimate of the spectrum $\Phi_{v}$ is to form a weighted sum

$$
\hat{\Phi}_{v}=\frac{1}{\rho} \hat{\mathcal{V}} \mathcal{W} \hat{\mathcal{V}}^{*}
$$

The question is, what should the normalization factor $\rho$ be? Consider the $i, j$ element of the matrix above and note that $\mathcal{Y} P_{u}=\mathcal{V} P_{u}$. Thus

$$
\mathrm{E} \hat{\Phi}_{v}^{i, j}=\frac{1}{\rho} \mathcal{V}_{i} P_{u} \mathcal{W} P_{u}^{*} \mathcal{V}_{j}^{*}=\frac{1}{\rho} \operatorname{trE} \mathcal{V}_{j}^{*} \mathcal{V}_{i} P_{u} \mathcal{W}
$$

Assuming $\Phi_{v}$ to be constant (at least over the interval covered by the weights), and that the frequency grid is such that $v\left(\omega_{k}\right)$ are uncorrelated (recall the comment below (1.24)) we have

$$
\mathrm{EV}_{j}^{*} \mathcal{V}_{i}=\Phi_{v}^{i, j} \cdot I_{N \times N}
$$

with the $N \times N$ identity matrix. Moreover

$$
\eta=\operatorname{tr} P_{u} \mathcal{W}=\operatorname{tr} \mathcal{W}-\operatorname{tr}\left(\mathcal{U} \mathcal{W} \mathcal{U}^{*}\right)^{-1} \mathcal{U} \mathcal{W}^{2} \mathcal{U}^{*}
$$

This shows that the correct normalization in (1.36) is $\rho=\eta$ (Note that in case equal weigths are used, that is, $\mathcal{W}=I$, then the normalization becomes the familiar $\rho=N-m$.)

This way (1.33) of estimating the frequency function is closely related to classical spectral analysis, since $\mathcal{U W U}^{*}$ can be seen as an estimate of the input spectral matrix and $\mathcal{Y W U}^{*}$ is an estimate of the cross spectrum. (See also Chapter 6 in [7].) The weights in $\mathcal{W}$ then correspond to the frequency (or tapering) windows, like the Bartlett, Parzen or Hamming windows typically applied in spectral analysis. Displaying the kinship to local polynomial modeling in curve fitting gives extra insight into the role of these windows. It also shows how to find the right normalization for unbiased estimation of the additive disturbance spectrum, which is important for narrow frequency windows. It may be easy to overlook the second term of (1.37). 
Nothing in this treatment requires that the window $\mathcal{W}$ is the same for all frequencies. On the contrary, it is natural to let the bandwidth depend on the actual frequency where $G$ is estimated. This is how the function spafdr (spectral analysis with frequency dependent resolution) in the SySTEm IDENTIFICATION Toolbox, [8], is implemented. A related approach to smoothing the ETFE is described in [12].

\subsection{Fitting the Frequency Function by Parametric Methods}

\subsubsection{The Model Structure}

A model structure for a linear system is simply a parameterization of the frequency function

$$
G(i \omega, \theta)
$$

possibly together with a parameterization of the additive noise spectrum

$$
\Phi_{v}(\omega, \theta)=H(i \omega, \theta) \Lambda(\theta) H^{*}(i \omega, \theta)
$$

where the second step shows the spectrum factorized using a monic, stable and inversely stable transfer function $H$.

The actual parameterization can be done in many different ways. The underlying description could be a discrete time ARMAX model

$$
A(q) y(t)=B(q) u(t)+C(q) e(t)
$$

with the coefficients of the polynomials (in $q^{-1}$ ) $A, B$ and $C$ comprising $\theta$. This gives

$$
\begin{aligned}
& G\left(e^{i \omega}, \theta\right)=\frac{B\left(e^{i \omega}\right)}{A\left(e^{i \omega}\right)} \\
& H\left(e^{i \omega}, \theta\right)=\frac{C\left(e^{i \omega}\right)}{A\left(e^{i \omega}\right)}
\end{aligned}
$$

Note the similarity with the basic forms (1.5) and (1.6) for $x=e^{-i \omega}$.

A physically parameterized state space model

$$
\begin{array}{ll}
\dot{x}(t)=A(\theta) x(t)+B(\theta) u(t)+w(t) ; & \mathrm{E} w(t) w^{T}(s)=Q(\theta) \delta(t-s) \\
y(t)=C(\theta) x(t)+D(\theta) u(t)+e(t) ; & \mathrm{E} e(t) e^{T}(s)=R(\theta) \delta(t-s)
\end{array}
$$


corresponds to

$$
\begin{aligned}
& G(i \omega, \theta)=C(\theta)(i \omega I-A(\theta))^{-1} B(\theta)+D(\theta) \\
& H(i \omega, \theta)=C(\theta)(i \omega I-A(\theta))^{-1} K(\theta)+I
\end{aligned}
$$

where $K(\theta)$ and $\Lambda(\theta)$ are computed from $A, C, Q$ and $R$ as the steady state Kalman filter's gain and innovations variance.

Many other types of parameterizations are of course possible.

\subsubsection{Frequency Domain Data}

Assume that the data are given in the frequency domain. We can then form the ETFE as described in Sections 1.3.2 and 1.3.3. The weighted least squares fit between the ETFE and the parameterized curve is then obtained as in (1.10):

$$
\begin{aligned}
V(\theta)= & \sum_{\ell=1}^{M} \operatorname{vec}\left(\hat{\hat{G}}\left(i \omega_{\ell}\right)-G\left(i \omega_{\ell}, \theta\right)\right)^{*}\left\{\left[\mathcal{U}_{N} \mathcal{U}_{N}^{*}\right] \otimes \Phi_{v}^{-1}\left(\omega_{\ell}\right)\right\} \\
& \times \operatorname{vec}\left(\hat{\hat{G}}\left(i \omega_{\ell}\right)-G\left(i \omega_{\ell}, \theta\right)\right)
\end{aligned}
$$

where the frequencies $\omega_{\ell}$ are from the grid (1.26). Here we have first formed a column vector from the matrix $\hat{\hat{G}}-G$ and then weighted with the inverse covariance matrix, (1.29), of the measurement noise at the frequency in question. (Note that $(A \otimes B)^{-1}=A^{-1} \otimes B^{-1}$.) Applying the formula

$$
\operatorname{vec}\left(D^{*}\right)^{*}\left(C^{*} \otimes A\right) \operatorname{vec}(B)=\operatorname{tr}(A B C D)
$$

now gives

$$
V(\theta)=\sum_{\ell=1}^{M} \operatorname{tr}\left[\hat{\hat{G}}\left(i \omega_{\ell}\right)-G\left(i \omega_{\ell}, \theta\right)\right]\left[\mathcal{U}_{N} \mathcal{U}_{N}^{*}\right]\left[\hat{\hat{G}}\left(i \omega_{\ell}\right)-G\left(i \omega_{\ell}, \theta\right)\right]^{*} \Phi_{v}^{-1}\left(\omega_{\ell}\right)
$$

which in view of (1.27) also can be written

$$
V(\theta)=\sum_{\ell=1}^{M} \operatorname{tr}\left[\mathcal{Y}_{N}\left(\omega_{\ell}\right)-G\left(i \omega_{\ell}, \theta\right) \mathcal{U}_{N}\left(\omega_{\ell}\right)\right]^{*} \Phi_{v}^{-1}\left(\omega_{\ell}\right)\left[\mathcal{Y}_{N}\left(\omega_{\ell}\right)-G\left(i \omega_{\ell}, \theta\right) \mathcal{U}_{N}\left(\omega_{\ell}\right)\right]
$$


The expression within bracket is a $p \times m$ matrix which means that we can go back to the original vectors $U^{(r)}\left(\omega_{\ell}\right)$ and $Y^{(r)}\left(\omega_{\ell}\right)$ in (1.25) to obtain

$$
\begin{aligned}
V(\theta)= & \sum_{\ell=1}^{M} \sum_{r=1}^{m}\left[Y_{N}^{(r)}\left(\omega_{\ell}\right)-G\left(i \omega_{\ell}, \theta\right) U_{N}^{(r)}\left(\omega_{\ell}\right)\right]^{*} \Phi_{v}^{-1}\left(\omega_{\ell}\right) \\
& \times\left[Y_{N}^{(r)}\left(\omega_{\ell}\right)-G\left(i \omega_{\ell}, \theta\right) U_{N}^{(r)}\left(\omega_{\ell}\right)\right]
\end{aligned}
$$

Here we have an $m$-fold repetition of frequencies on the courser grid (1.26). By a small approximation we can move to the finer grid (1.24) and obtain

$$
V(\theta)=\sum_{k=1}^{N}\left[Y_{N}\left(\omega_{k}-G\left(i \omega_{k}, \theta\right) U_{N}\left(\omega_{k}\right)\right]^{*} \Phi_{v}^{-1}\left(\omega_{k}\right)\left[Y_{N}\left(\omega_{k}\right)-G\left(i \omega_{k}, \theta\right) U_{N}\left(\omega_{k}\right)\right]\right.
$$

\subsubsection{Prediction Errors From Time Domain Data}

If we start with time domain data (1.17) we could of course directly transform to frequency domain data and go through steps of the previous subsection. It is instructive to follow the calculations directly in the time domain.

The discrete time domain version of the model (1.38), (1.39) is

$$
y(t)=G(q, \theta) u(t)+H(q, \theta) e(t)
$$

For equidistantly sampled data we can form the prediction errors for this model as

$$
\varepsilon(t, \theta)=H^{-1}(q, \theta)(y(t)-G(q, \theta) u(t))
$$

Assuming that these have covariance matrix $\Lambda$, a natural criterion to minimize (which equals the maximum likelihood criterion if $\Lambda$ is known and $e$ is Gaussian) is

$$
V(\theta)=\sum_{k=1}^{N} \varepsilon^{T}(t, \theta) \Lambda^{-1} \varepsilon(t, \theta)
$$

Applying Parseval's relationship to (1.48), (1.47) and ignoring transient effects (or assuming periodic data) now gives for this criterion

$$
\begin{aligned}
V(\theta) & =\sum_{k=1}^{N}\left[Y_{N}\left(\omega_{k}\right)-G\left(i \omega_{k}, \theta\right) U_{N}\left(\omega_{k}\right)\right]^{*} \Phi_{v}^{-1}\left(\omega_{k}, \theta\right) \\
& \times\left[Y_{N}\left(\omega_{k}\right)-G\left(i \omega_{k}, \theta\right) U_{N}\left(\omega_{k}\right)\right] \\
\Phi_{v}(\omega, \theta) & =H(i \omega, \theta) \Lambda H^{*}(i \omega, \theta)
\end{aligned}
$$


This is the same as (1.45) and we can now track back to the curve fitting expression between the ETFE and the parameterized curve in (1.40). Even in the time domain, multi-variable case the basic methods consequently still are curve fitting. We have also displayed the nature of the noise model in (1.46): It just provides the weighting in this fit.

\subsection{Conclusions}

Phrasing standard methods for linear system identification as curve fitting brings out several common features and gives some additional insight. It also shows that the bottom line in identification is quite simple and relies upon early work in statistics.

The subspace methods were not explicitly discussed in this contribution. The link to curve fitting is conceptually as follows: The first step in subspace methods is to form a big Hankel matrix from observed data. This can be seen as a high order ARX model for the system. The second step is to approximate this matrix with a low rank one using SVD. This is basically related to Hankel norm approximation of high order systems by lower order ones, which in turn can be interpreted as approximating the corresponding frequency functions, see [4]. 


\section{Bibliography}

[1] D.R. Brillinger. Time Series: Data Analysis and Theory. Holden-Day, San Francisco, 1981.

[2] W.S. Cleveland and E. Grosse. Computational methods for local regression. Statistics and Computing, 1:47-62, 1991.

[3] J. Fan and I. Gijbels. Local Polynomial Modelling and Its Applications. Number 66 in Monographs on Statistics and Applied Probability. Chapman \& Hall, 1996.

[4] K. Glover. All optimal Hankel norm approximations of linear multivariable systems and their L-infinity error bounds. Int. Journal of Control, 39:1115-1193, 1984.

[5] G.M. Jenkins and D.G. Watts. Spectral Analysis. Holden-Day, San Francisco, 1968.

[6] W. E. Larimore. Canonical variate analysis in identification, filtering and adaptive control. In Proc. 29th IEEE Conference on Decision and Control, pages 596-604, Honolulu, Hawaii, December 1990.

[7] L. Ljung. System Identification - Theory for the User. Prentice-Hall, Upper Saddle River, N.J., 2nd edition, 1999.

[8] L. Ljung. System Identification Toolbox for use with MatLAB. Version 6. The MathWorks, Inc, Natick, MA, 6th edition, 2002.

[9] E. Nadaraya. On estimating regression. Theory of Prob. and Applic., 9:141-142, 1964.

[10] J. Roll, A. Nazin, and L. Ljung. A non-asymptotic approach to local modelling. In Proc. IEEE Conf. on Decision and Control, Las Vegas, NV, Dec 2002. To appear. 
[11] T. Söderström and P. Stoica. System Identification. Prentice-Hall Int., London, 1989.

[12] A. Stenman, F. Gustafsson, D.E. Rivera, L. Ljung, and T. McKelvey. On adaptive smoothing of empirical transfer function estimates. Control Engineering Practice, 8:1309-1315, Nov 2000.

[13] C.J. Stone. Consistent non-parametric regression (with discussion). Ann. Statist., 5:595-645, 1977.

[14] P. Van Overschee and B. DeMoor. Subspace Identification of Linear Systems: Theory, Implementation, Applications. Kluwer Academic Publishers, 1996.

[15] M. Verhaegen. Identification of the deterministic part of MIMO state space models, given in innovations form from input-output data. Automatica, 30(1):61-74, January 1994. 
Abstract

Keywords: 


Avdelning, Institution
Division, Department
Division of Automatic Control
Department of Electrical Engineering

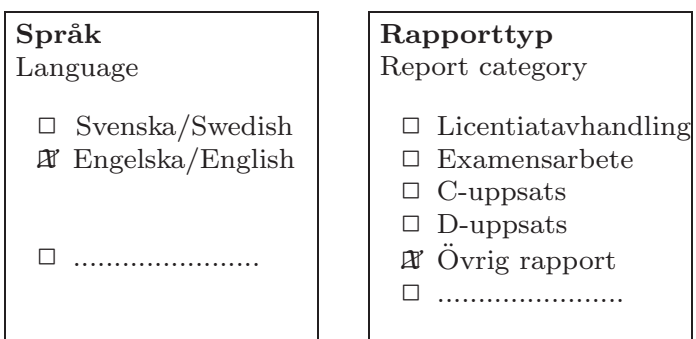

URL för elektronisk version

http://www. control.isy.liu.se

\begin{tabular}{|c|c|}
\hline ISBN & \\
\hline ISRN & \\
\hline $\begin{array}{l}\text { Serietitel och serienummer } \\
\text { Title of series, numbering }\end{array}$ & ISSN \\
\hline LiTH-ISY-R-2466 & \\
\hline
\end{tabular}

Titel

Title Linear System Identification as Curve Fitting

Författare

Author Lennart Ljung, ,

Sammanfattning

Abstract

Nyckelord

Keywords 